\title{
A new tool for detection of type I interferon activation in systemic lupus erythematosus
}

\author{
Kyriakos A Kirou* and George D Kalliolias \\ See related research by Li et al., http://arthritis-research.com/content/12/3/R90
}

\begin{abstract}
The IFN-I pathway is activated in systemic lupus erythematosus (SLE) and appears to be important in the pathogenesis of the disease. As a result, several clinical trials of anti-IFN monoclonal antibodies, which hold promise to control the disease, have been launched. Additionally, activation of IFN-I might be important in the prognosis and activity assessment of the disease. Therefore, new biomarkers that reflect activity of the IFN-I pathway and are simple to measure, such as the monocyte CD64 receptor, are expected to have a great impact on the management of SLE, if properly validated.
\end{abstract}

Over the past decade, numerous publications have stressed the importance of IFN-I in the pathogenesis of systemic lupus erythematosus (SLE), but their assays typically used microarrays and/or quantitative PCR (qPCR) and thus appear to be laborious and not well suited for use in clinical practice. In the previous issue of Arthritis Research Therapy, Li and colleagues [1] have investigated a much simpler methodology to measure the level of activation of IFN-I in patients with SLE. They measured the expression of CD64 (FcyRI) by flow cytometry on monocytes and demonstrated high levels in SLE compared with healthy controls. The authors also showed that CD64 levels correlated with IFN-stimulated gene (ISG) expression (by qPCR) and disease activity (by Systemic Lupus Erythematosus Disease Activity Index (SLEDAI)) and that CD64 was downregulated in four patients who received pulse methylprednisolone therapy. As expected, CD64 was induced in normal monocytes by IFN-I in vitro. Similar findings have been obtained in SLE monocytes by flow cytometry in another study focused

*Correspondence: kirouk@hss.edu

Mary Kirkland Center for Lupus Research, Hospital for Special Surgery, 535 East 70th Street, New York, NY 10021, USA on sialoadhesin (Siglec-1 or CD169), although this assay required indirect immunofluorescence staining [2]. Both these molecules appear promising as biomarkers of IFN-I activation in SLE.

The clinical significance of IFN-I pathway activation in SLE is multifaceted. First, the pathway has been implicated in the pathogenesis of the disease, and therefore targeted therapies against IFN-I are currently in clinical trials. Second, IFN-I activation may identify a subset of SLE patients with potential diagnostic, prognostic and therapeutic implications. Third, change in IFN-I activity levels may reflect change in disease activity and thus help clinical management of the disease. In this context, the data by $\mathrm{Li}$ and colleagues on CD64 expression have clinical relevance as they might facilitate research in all of the above areas with a simple tool.

The implication of IFN-I in SLE pathogenesis comes from multiple pieces of evidence, including genetic, gene expression association studies, and induction of SLE by therapeutic administration of IFN-I [3]. The potential mechanisms by which IFN-I may promote inflammation and autoimmunity have been reviewed recently and include activation of immature dendritic cells and break of peripheral tolerance, augmentation of humoral immunity pathways with production of pathogenic antibodies, induction of Th1 cells, chemokine production, and priming of myeloid cells for enhanced responses to inflammatory stimuli [4]. The emerging evidence about the pathogenic role of IFN-I in SLE led to the recent introduction in clinical trials of several anti-IFN antibodies. This exciting new era of IFN-I targeting should greatly benefit from simple biomarkers of the pathway's activity, such as CD64 and CD169 expression. This would facilitate stratification of SLE patients according to IFN-I activation, as well as monitoring of the degree of IFN-I inhibition during therapy - adequate to suppress disease activity, but not excessive to cripple immunosurveillance.

Cross-sectional studies, including our own and the study by $\mathrm{Li}$ and colleagues, have shown that IFN-I activation in SLE is present in about half of adult patients and it is associated with disease activity, renal involvement, as well as autoantibodies to dsDNA, and RNA 
Binding Proteins (anti-Ro, La, Sm, RNP) [1,5-7]. This group of patients might represent a different subclass of the disease where the IFN-I pathway is dominant and therefore its therapeutic targeting most beneficial. The design of those studies, however, leaves open the possibility that some patients may be positive for IFN only intermittently, especially during disease flares, and again negative after aggressive therapy, such as with pulse methylprednisolone [1,5]. More research needs to be done in this area before conclusions can be drawn. We believe that although SLE patients should be stratified in clinical trials of anti-IFN therapy, patients negative for IFN-I activation should not be excluded from the trials.

Two longitudinal studies failed to show ability of IFN-I activation to parallel acute changes of disease activity $[6,7]$. However, one of those studies used microarray data (which is less accurate than qPCR) for their ISG score [7] and both had only few patients with more than two visits. Moreover, IFN-regulated chemokine levels did parallel disease activity in a larger study [8]. Interestingly, high baseline levels of these chemokines substantially increased the risk for lupus flare, especially a renal one, in the next year [8]. Flares were also increased for patients with high baseline ISG scores, but in a more delayed manner [6]. In our experience, about 30 to $40 \%$ of patients, followed longitudinally for at least 2 years, demonstrate parallel courses of SLEDAI and ISG scores [9]. Based on the above studies, it appears that the matter has not been resolved yet, but it is possible that ISG scores work as biomarkers of disease activity only in a subgroup of patients, and not necessarily the ones with high baseline IFN-I activity. Other pathway signatures or a combination of those might be eventually required to evaluate all patients [10].

Although measurement of monocyte CD64 and CD169 expression appears promising, it is likely not specific for either IFN-I or SLE disease activity. Similar to other ISGs, both can be induced by viral infection and IFN- $\gamma$, whereas CD64 expression may also be induced by IL-10 $[1,11,12]$. Thus, at this point, none of the above gene measurements can be expected to substitute for clinical judgment to differentiate lupus flare from infection. Furthermore, before these new monocyte cell surface markers can fulfill their promise for ease and efficiency of IFN-I detection, they will need to be validated against currently used IFN-I molecular assays (especially qPCR) in carefully conducted large longitudinal prospective studies of SLE patients.

\section{Abbreviations}

IFN = interferon; ISG = IFN-stimulated gene; $\mathrm{PPCR}=$ quantitative PCR; SLE = systemic lupus erythematosus; SLEDAI = Systemic Lupus Erythematosus Disease Activity Index.

\section{Competing interests}

KAK has applied for a patent for an interferon assay. GDK declares that he has no competing interests.

Published: 26 August 2010

References

1. Li Y, Lee PY, Kellner ES, Paulus M, Switanek J, Xu Y, Zhuang H, Sobel ES, Segal MS, Satoh M, Reeves WH: Monocyte surface expression of Fcy receptor RI (CD64), a biomarker reflecting type-I interferon levels in systemic lupus erythematosus. Arthritis Res Ther 2010, 12:R90.

2. Biesen R, Demir C, Barkhudarova F, Grün JR, Steinbrich-Zöllner M, Backhaus M, Häupl T, Rudwaleit M, Riemekasten G, Radbruch A, Hiepe F, Burmester GR, Grützkau A: Sialic acid-binding lg-like lectin 1 expression in inflammatory and resident monocytes is a potential biomarker for monitoring disease activity and success of therapy in systemic lupus erythematosus. Arthritis Rheum 2008, 58:1136-1145.

3. Kirou KA, Mavragani CP, Crow MK: Activation of type I interferon in systemic lupus erythematosus. Expert Rev Clin Immunol 2007, 3:579-588.

4. Kalliolias GD, Ivashkiv LB: Overview of the biology of type I interferons. Arthritis Res Ther 2010, 12 Suppl 1:S1.

5. Kirou KA, Lee C, George S, Louca K, Peterson MG, Crow MK: Activation of the interferon-alpha pathway identifies a subgroup of systemic lupus erythematosus patients with distinct serologic features and active disease. Arthritis Rheum 2005, 52:1491-1503.

6. Landolt-Marticorena C, Bonventi G, Lubovich A, Ferguson C, Unnithan T, Su J, Gladman DD, Urowitz M, Fortin PR, Wither J: Lack of association between the interferon-alpha signature and longitudinal changes in disease activity in systemic lupus erythematosus. Ann Rheum Dis 2009, 68:1440-1446.

7. Petri M, Singh S, Tesfasyone H, Dedrick R, Fry K, Lal P, Williams G, Bauer J, Gregersen P, Behrens T, Baechler E: Longitudinal expression of type I interferon responsive genes in systemic lupus erythematosus. Lupus 2009, 18:980-989.

8. Bauer JW, Petri M, Batliwalla FM, Koeuth T, Wilson J, Slattery C, PanoskaltsisMortari A, Gregersen PK, Behrens TW, Baechler EC: Interferon-regulated chemokines as biomarkers of systemic lupus erythematosus disease activity: a validation study. Arthritis Rheum 2009, 60:3098-3107.

9. Barillas-Arias L, MacDermott EJ, Duculan R, Santiago A, Gordon E, Cole P, Crow MK, Kirou KA: Longitudinal prospective study of type I interferon pathway activation as a biomarker of disease activity in patients with systemic lupus erythematosus (SLE) - Interim analysis. Arthritis Rheum 2008, 56:4245.

10. Chaussabel D, Quinn C, Shen J, Patel P, Glaser C, Baldwin N, Stichweh D, Blankenship D, Li L, Munagala I, Bennett L, Allantaz F, Mejias A, Ardura M, Kaizer E, Monnet L, Allman W, Randall H, Johnson D, Lanier A, Punaro M, Wittkowski KM, White P, Fay J, Klintmalm G, Ramilo O, Palucka AK, Banchereau J, Pascual V: A modular analysis framework for blood genomics studies: application to systemic lupus erythematosus. Immunity 2008, 29:150-164.

11. te Velde AA, de Waal Malefijt R, Huijbens RJ, de Vries JE, Figdor CG: IL-10 stimulates monocyte Fc gamma R surface expression and cytotoxic activity. Distinct regulation of antibody-dependent cellular cytotoxicity by IFN-gamma, IL-4, and IL-10. J Immuno/ 1992, 149:4048-4052.

12. Rempel H, Calosing C, Sun B, Pulliam L: Sialoadhesin expressed on IFNinduced monocytes binds HIV-1 and enhances infectivity. PLoS One 2008, 3:e1967.

doi:10.1186/ar3114

Cite this article as: Kirou KA, Kalliolias GD: A new tool for detection of type I interferon activation in systemic lupus erythematosus. Arthritis Research \& Therapy 2010, 12:138. 